\title{
过渡金属催化氮杂二环烯烃不对称开环反应的研究进展
}

\author{
程汉超梁秀丽李晓璐龙玉华杨定乔* \\ (华南师范大学化学与环境学院 广州 510006)
}

\begin{abstract}
摘要 综述了近年来过渡金属催化氮杂二环烯烃的开环反应的研究进展, 重点讨论了过渡金属催化剂的种类、亲核试 剂的类型、配体、底物氮杂二环烯烃及其衍生物的结构、溶剂和添加剂等因素对开环反应的影响，并对开环反应的可 能的机理进行了讨论.
\end{abstract}

关键词 氮杂二环烯烃; 过渡金属; 催化; 开环反应

\section{Progress in Transition Metal-Catalyzed Asymmetric Ring-Opening Reactions of Azabicyclic Alkenes}

\author{
Cheng, Hanchao Liang, Xiuli Li, Xiaolu Long, Yuhua Yang, Dingqiao*
}

(School of Chemistry and Environment, South China Normal University, Guangzhou 510006)

\begin{abstract}
The recent progress in transition metal-catalyzed ring-opening reactions of azabicyclic alkenes was reviewed focused on the influence of the types of transition metal catalysts, nucleophiles, ligands, the structures of azabicyclic alkenes and its derivatives, solvents and additives on the ring-opening reactions. Moreover, the possible mechanisms of the ring-opening reactions were also discussed.
\end{abstract}

Keywords azabicyclic alkenes; transition metal; catalyze; ring-opening reaction

在自然界中，四氢化荟结构单元是具有生物活性的 化合物中常见的骨架之一，许多肾上腺激素、抗生素以 及中国紫杉醇中的异紫杉脂素、去氧鬼自素葡萄糖酯苷 等化合物中都含有四氢化芸的基本结构. 这些化合物具 有广泛的生理活性，如抗抑郁、抗焦虑、消炎止痛、抗 帕金森、抗癌等 ${ }^{[1 \sim 1]}$. 过渡金属催化氮杂二环烯烃的不 对称开环反应是一种高对映选择性地合成此类药物中 间体的方法，如 Eq. 1 所示:

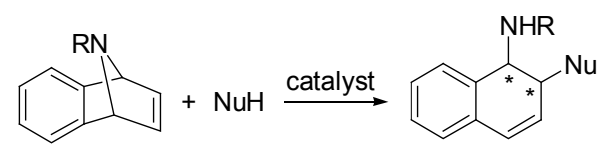

此类反应只需一步就可生成具有两个手性中心的 二氢化菜骨架, 在合适的过渡金属催化剂催化下, 开环 反应会产生一种高对映选择性的产物. 同时催化剂的活 性对反应的速率、产率、立体选择性、区域选择性和化 学选择性也有着重要的影响 ${ }^{[12,13]}$. 由于这类反应具有低

催化剂用量、高产率和高对映选择性等特点，化学家们 已对此产生了浓厚的兴趣 ${ }^{[14]}$, 并取得了很好的研究成 果. 综述了近年来 $\mathrm{Ni}, \mathrm{Cu}, \mathrm{Ru}, \mathrm{Rh}, \mathrm{Ir}$ 和 $\mathrm{Pd}$ 过渡金属催化 氮杂二环烯烃开环反应的研究进展.

\section{1 镍催化氮杂二环烯烃的开环反应}

\section{1 有机碘试剂作为亲核试剂}

1999 年, Cheng 等 ${ }^{[15]}$ 以 $\mathrm{Ni}\left(\mathrm{PPh}_{3}\right)_{2} \mathrm{Cl}_{2}$ 作催化剂和有 机碘作为亲核试剂, 在锌粉存在下, 以 $\mathrm{CH}_{3} \mathrm{CN}$ 为溶剂, 实现了氮杂二环烯烃的开环反应(Eq. 2). 研究表明: 过 量的配体 $\mathrm{PPh}_{3}$ 会抑制催化剂的活性，阻碍反应的进行. 他们还尝试了不同结构的有机碘作为亲核试剂，反应具

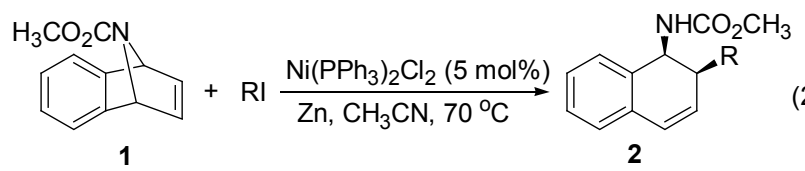

\footnotetext{
*E-mail: yangdq@scnu.edu.cn

Received June 22, 2011; revised September 30, 2011; accepted October 25, 2011.

Project supported by the National Natural Science Foundation of China (No. 21172081) and the Natural Science Foundation of Guangdong Province (No. 8251063101000002)

国家自然科学基金(No. 21172081)和广东省自然科学基金(No. 8251063101000002)资助项目.
} 
有较好的立体选择性, 得到的产物为 $s y n$ 构型, 产率高 达 97\%(表 1).

表 1 亲核试剂对镍催化氮杂二环烯烃开环反应的影响

Table 1 Effect of nucleophiles on the nickel-catalyzed ringopening reactions of azabicyclic alkenes

\begin{tabular}{clcc}
\hline Entry & \multicolumn{1}{c}{$\mathrm{RI}$} & Time/h & Yield/\% \\
\hline 1 & $\mathrm{C}_{6} \mathrm{H}_{5} \mathrm{I}$ & 1.4 & 78 \\
2 & $o-\mathrm{CH}_{3} \mathrm{C}_{6} \mathrm{H}_{4} \mathrm{I}$ & 2.0 & 78 \\
3 & $p-\mathrm{CH}_{3} \mathrm{C}_{6} \mathrm{H}_{4} \mathrm{I}$ & 3.0 & 97 \\
4 & $m-\mathrm{ClC}_{6} \mathrm{H}_{4} \mathrm{I}$ & 1.5 & 85 \\
5 & $o-\mathrm{C}_{10} \mathrm{H}_{7} \mathrm{I}$ & 12 & 83 \\
\hline
\end{tabular}

Cheng 等还提出了该反应的机理(Scheme 1): $\mathrm{Ni}$ 金 属催化剂被 $\mathrm{Zn}$ 还原后再与亲核试剂结合, 形成了热不 稳定的中间体 $\mathrm{Ni}\left(\mathrm{PPh}_{3}\right)_{2} \mathrm{RI}$; 此中间体分解出 $\mathrm{PPh}_{3}$, 产生 了配位空位, 再与底物 $\mathbf{1}$ 反应得到了中间体 $\mathbf{3}$, 此步反 应是决定整个催化反应速度的步骤; $\beta$ 位杂原子消去后, 再在金属 $\mathrm{Zn}$ 还原作用下，一方面生成化合物 2 的锌盐, 锌盐化合物水解后就可以得到顺式产物 2, 另一方面生 成了 $\mathrm{Ni}(0)$ 催化剂. $\mathrm{Ni}(0)$ 催化剂再与 $\mathrm{RI}$ 反应, 整个催化 反应得以循环进行.

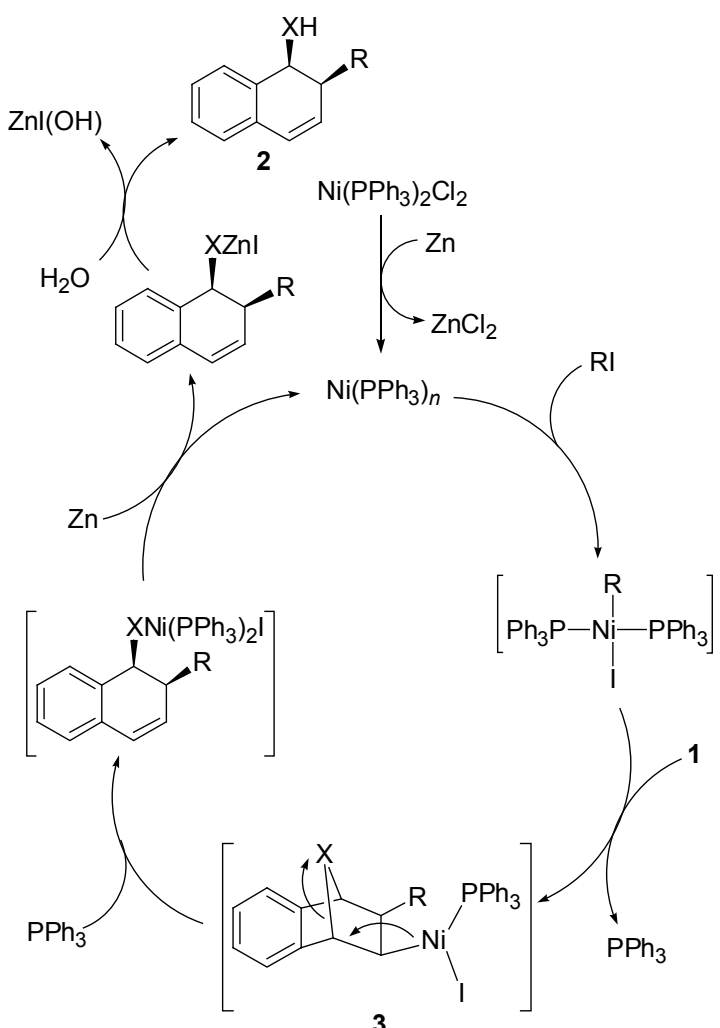

Scheme 1

2003 年, Cheng 等 ${ }^{[16]}$ 在研究 $\mathrm{Ni}$ 催化氧杂二环烯烃与 有机碘试剂进行开环加成的过程中, 将研究所得最佳实
验条件应用于氮杂二环烯烃开环反应(Eq. 3)，结果发现， 当配体为 1,2-二(二苯基膦)乙烷(dppe)时，氮杂二环烯烃 开环并生成一种内酰胺 5, 产率为 $80 \%$.


\section{2 末端炔烃类化合物作为亲核试剂}

2002 年 Cheng 等 ${ }^{[17]}$ 使用末端炔烃作为亲核试剂进 行开环反应，得到顺式开环产物(Eq. 4). 首先他们探讨 了不同 $\mathrm{R}$ 基的末端炔烃的对开环反应的影响，在 $\mathrm{Ni}$ (dppe) $\mathrm{Cl}_{2}$ 催化下, 无论是脂肪族末端炔烃还是芳香族 末端炔烃都能实现开环反应，同时溶剂对开环反应影响 较大，选择甲苯作溶剂最有利于开环反应，反应中加入 锌粉能加快反应速率，加入路易斯酸 $\mathrm{ZnCl}_{2}$ 能提高产率 $(92 \%)$.

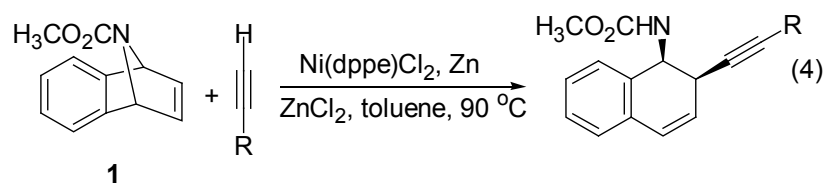

2003 年, Cheng 等 ${ }^{[18]}$ 还以炔烃酸酯类化合物作为亲 核试剂，实现了氮杂二环烯烃的开环反应(Eq. 5). 他们 尝试了多种 $\mathrm{Ni}$ 催化剂, 发现 $\mathrm{Ni}(\mathrm{dppe}) \mathrm{Br}_{2}$ 对开环反应最 有利，溶剂的选择对开环反应极为关键，开环反应以 $\mathrm{CH}_{3} \mathrm{CN}$ 作为溶剂, 反应效果最好, 得到 $s y n$ 构型的产物, 产率可达 78\%.

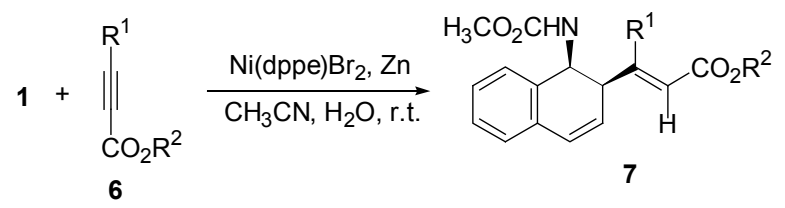

Cheng 等还提出了该反应的机理(Scheme 2): Ni(II) 催化剂在锌粉的还原作用下生成了 $\mathrm{Ni}(0)$ 催化剂; 底物 1 和亲核试剂 6 在 $\mathrm{Ni}(0)$ 催化剂参与下, 发生有区域选择性 地偶联反应，产生了中间体 $\mathbf{8}$; 随着 $\beta$ 位杂原子消去和 质子化，反应得到了最终的产物 7. 值得注意的是：该 反应和 Cheng 等此前报道的反应 ${ }^{[16]}$ 都会产生中间体 8 和 9 , 在质子存在的条件下，中间体最终会转化成产物 7, 当没有质子时，中间体 9 就会经过重排、成环得到化 合物 5 的衍生物. 


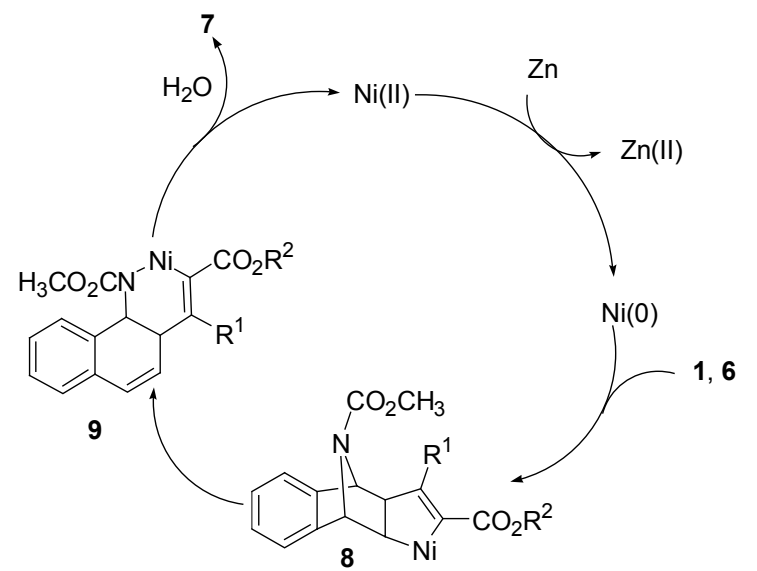

Scheme 2

\section{3 乙烯基锆类化合物作为亲核试剂}

2004 年, Cheng 等 ${ }^{[19]}$ 以乙烯基锆类化合物作为亲核 试剂. 研究表明: 在无锌粉存在时, 开环反应产率很低, 但加入很少量的锌粉就能大大提高产率, 开环反应得到 $s y n$ 构型的产物(Eq. 6), 产率可达 83\%.



2003 年, Cheng 等 ${ }^{[20]}$ 还报道了在有机酸和锌粉存在 下, 以 $\mathrm{Ni}(\mathrm{BINAP}) \mathrm{Cl}_{2}$ 和 $\mathrm{Pd}(\mathrm{BINAP}) \mathrm{I}_{2}$ 催化氮杂二环烯烃 的开环反应(Eq. 7). 反应可得到 $89 \%$ 的产率和 $90 \% e e$,

且 $\mathrm{Ni}$ 和 $\mathrm{Pd}$ 催化开环反应的机理 (Scheme 3)类似. 然而 值得一提的是, Cheng 等提出的反应机理与 Lautens 等 ${ }^{[21]}$ 在 2001 年报道的铑催化氧杂二环烯烃的反应机理不同: 后者的羧酸作为亲核试剂参与反应，而前者的䍨酸在反 应体系中只起着提供质子的作用，且不同的羧酸也能影 响 $e e$ 值大小.



\section{2 铜催化氮杂二环烯烃的开环反应}

\section{1 有机金属化合物作为亲核试剂}

2005 年, Arrayás 等 ${ }^{[22]}$ 报道了以格氏试剂(Grignard reagent)作为亲核试剂, 铜催化氮杂二环烯烃的开环反 应(Eq. 8). 反应具有较好的立体选择性, 产物以 anti 构 型为主. 他们发现: $\mathrm{N}$ 上取代基对开环反应影响极大, 取代基 $\mathrm{R}$ 为(2-pyridyl)sulfonyl 时对开环反应最有利，使 用 $\mathrm{CuCN}$ 作为催化剂, 能使反应活性更高. 他们还探讨 了不同格氏试剂对反应的影响(表 2). Arrayás 等 ${ }^{[23]}$ 在 2006 年再一次报道了铜催化氮杂二环烯烃的开环反应, 所得结果与他们在 2005 年 ${ }^{[22]}$ 报道的结果相同.

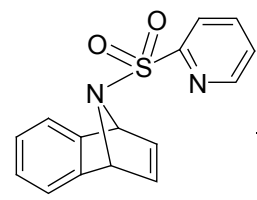

15

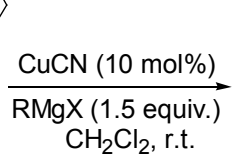
$\mathrm{CH}_{2} \mathrm{Cl}_{2}$, r.t.

(<smiles>[R]C1C=Cc2ccccc2C1NS(=O)O[Na]</smiles>

16

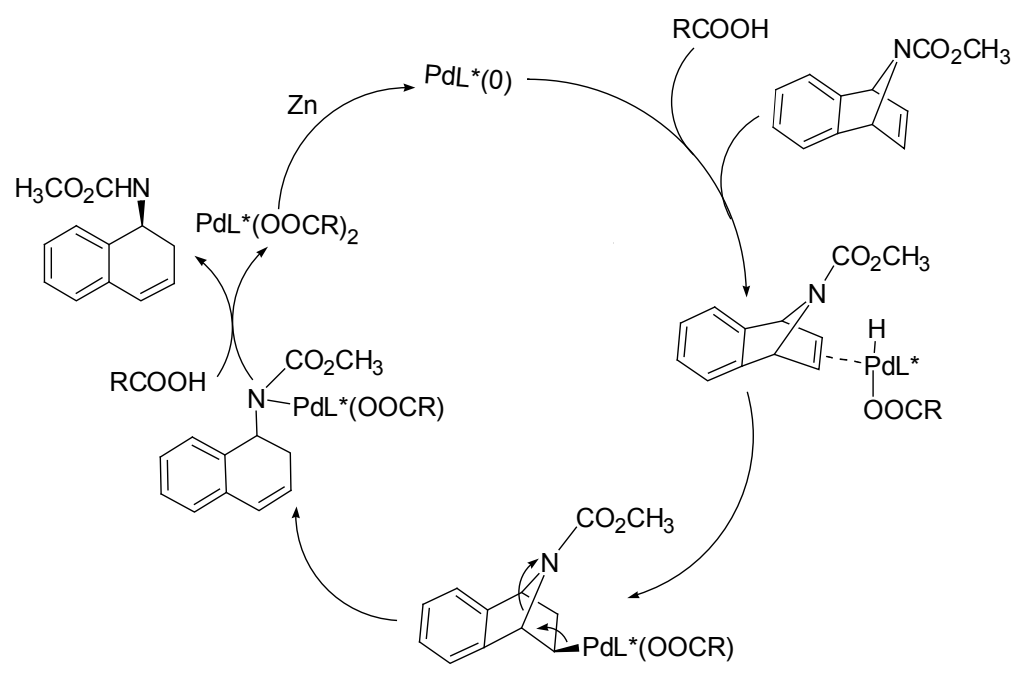

Scheme 3 
表 2 格氏试剂对 $\mathrm{Cu}$ 催化氮杂二环烯烃的开环反应的影响

Table 2 Effect of Grignard reagents on $\mathrm{Cu}$-catalyzed ringopening reactions of azabicyclic alkenes

\begin{tabular}{cclccc}
\hline Entry & $\mathrm{X}$ & \multicolumn{1}{c}{$\mathrm{R}$} & Time/min & anti/syn & Yield/\% \\
\hline 1 & $\mathrm{Br}$ & $\mathrm{Me}$ & 120 & $98: 2$ & 89 \\
2 & $\mathrm{Br}$ & $\mathrm{TMSCH}$ & 95 & $93: 7$ & 73 \\
3 & $\mathrm{Cl}$ & $\mathrm{Et}$ & 20 & $>98:<2$ & 65 \\
4 & $\mathrm{Cl}$ & $\mathrm{PhCH}$ & 20 & $>98:<2$ & 53 \\
5 & $\mathrm{Br}$ & $\mathrm{Ph}$ & 15 & $>98:<2$ & 93 \\
6 & $\mathrm{Cl}$ & $\mathrm{Tol}$ & 10 & $>98:<2$ & 91 \\
7 & $\mathrm{Cl}$ & $p-\mathrm{MeOC}_{6} \mathrm{H}_{4}$ & 10 & $>98:<2$ & 98 \\
8 & $\mathrm{Cl}$ & $p-\mathrm{FC}_{6} \mathrm{H}_{4}$ & 20 & $>98:<2$ & 92 \\
\hline
\end{tabular}

2005 年, Pineschi 等 ${ }^{[24]}$ 使用 $\mathrm{Cu}(\mathrm{OTf})_{2}$ 和 N-P 配体 $\mathbf{1 7}$ 催化二氮杂二环烯烃 18a, 18b 得到开环产物环戊烯 19a,

19b (Scheme 4). 他们尝试了不同的二烷基锌、三烷基铝 作为亲核试剂, 发现三烷基铝的活性更高, 其中使用 $\mathrm{Me}_{3} \mathrm{Al}$ 的反应转化率大于 $98 \%, e e$ 值达到 $86 \%$ (表 3).<smiles>O=C1C(c2ccccc2)C(=O)N2CCCN1C2</smiles>

18a

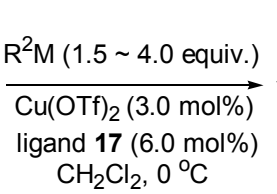

$\mathrm{CH}_{2} \mathrm{Cl}_{2}, 0^{\circ} \mathrm{C}$<smiles>CC(C)(C)c1cccc2c(=O)n3n(c(=O)c12)C1C=CC3C1</smiles>

$18 b$

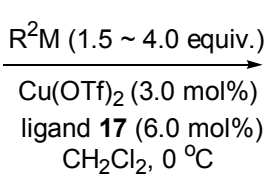
$\mathrm{CH}_{2} \mathrm{Cl}_{2}, 0{ }^{\circ} \mathrm{C}$

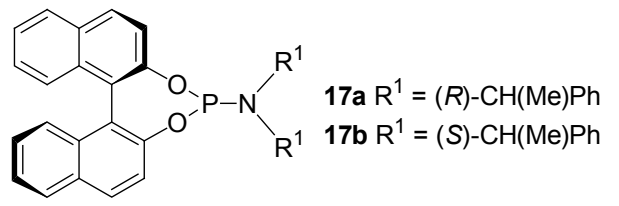

\section{Scheme 4}

表 3 有机金属试剂对 $\mathrm{Cu}$ 催化二氮杂二环烯烃的开环反应的 影响 ${ }^{a}$

Table 3 Effect of organometallic reagents on $\mathrm{Cu}$-catalyzed ring-opening reactions of diazabicyclic alkenes

\begin{tabular}{cclccl}
\hline Entry & Substrate & \multicolumn{1}{c}{$\mathrm{R}^{2} \mathrm{M}$} & Time/h & Conv./\% & $e e / \%$ \\
\hline 1 & $\mathbf{1 8 a}$ & $\mathrm{Et}_{2} \mathrm{Zn}$ & 24 & 38 & 3 \\
2 & $\mathbf{1 8 b}$ & $\mathrm{Et}_{2} \mathrm{Zn}$ & 6 & 22 & 0 \\
3 & $\mathbf{1 8 b}$ & $\mathrm{Bu}_{2} \mathrm{Zn}$ & 6 & 34 & 18 \\
4 & $\mathbf{1 8 a}$ & $\mathrm{Et}_{3} \mathrm{Al}$ & 4 & $>98$ & $66(+)$ \\
5 & $\mathbf{1 8 a}$ & $\mathrm{Me}_{3} \mathrm{Al}$ & 1 & $>98$ & $80(+)$ \\
6 & $\mathbf{1 8 b}$ & $\mathrm{Et}_{3} \mathrm{Al}$ & 4 & 94 & $78(+)$ \\
7 & $\mathbf{1 8 b}$ & $\mathrm{Me}_{3} \mathrm{Al}$ & 4 & $>98$ & $86(+)$ \\
$8^{b}$ & $\mathbf{1 8 b}$ & $\mathrm{Me}_{3} \mathrm{Al}$ & 4 & $>98$ & $64(-)$ \\
$9^{c}$ & $\mathbf{1 8 b}$ & $(n-\mathrm{Pr})_{3} \mathrm{Al}$ & 20 & $>98$ & $54(+)$ \\
$10^{c}$ & $\mathbf{1 8 b}$ & $(i-\mathrm{Bu})_{3} \mathrm{Al}$ & 20 & 95 & $14(-)$ \\
\hline
\end{tabular}

${ }^{a}$ Reactions entirely carried out at $0{ }^{\circ} \mathrm{C}$ with chiral ligand $(R, R, R)-\mathbf{1 7 a} .{ }^{b}$ Reaction carried out with diastereoisomeric ligand $(R, S, S)-\mathbf{1 7} \mathbf{b}$. ${ }^{c}$ Reaction carried out at up to room temperature.

\section{3 钉催化氮杂二环烯烃的开环反应}

\section{1 炔烃类化合物作为亲核试剂}

2006 年, $\mathrm{Tam}$ 等 ${ }^{[25]}$ 首次报道了 $\mathrm{Cp} \mathrm{p}^{*} \mathrm{Ru}(\mathrm{cod}) \mathrm{Cl}$ 催化氮 杂二环烯烃的开环反应(Eq. 9). 他们研究发现, $N$ 上取 代基 $\mathrm{R}$ 在开环反应中起着非常重要的作用，当 $\mathrm{R}$ 为 $\mathrm{CO}_{2} \mathrm{Me}$ 或 $\mathrm{CO}_{2} \mathrm{Bu}$ 时，反应不能发生，而只有当 $\mathrm{R}$ 为 $\mathrm{Ts}$ 时反应才能顺利进行，产率可达 $86 \%$.

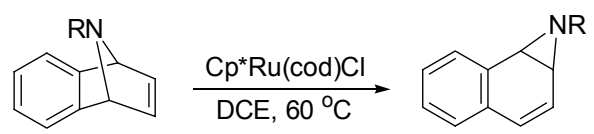

2007 年, Tam 等 ${ }^{[26]}$ 再次报道了 $\mathrm{Ru}$ 催化氮杂二环烯 烃的反应(Eq. 10). 反应在 $\mathrm{THF}$ 溶剂中、 $\mathrm{Cp} * \mathrm{Ru}(\mathrm{cod}) \mathrm{Cl}$ 催化条件下, 分别得到开环产物 21 和[2+2]环加成产物 22(表 4), 这就是一种高对映选择性地合成苯并吲哚衍 生物的方法. Tam 等还尝试无苯并环的氮杂二环烯烃 23 与炔烃 20 在同样的催化条件下反应，也能得到无苯并 环结构的开环产物 24, 但是没有环加成产物 25 生成 (Eq. 11)

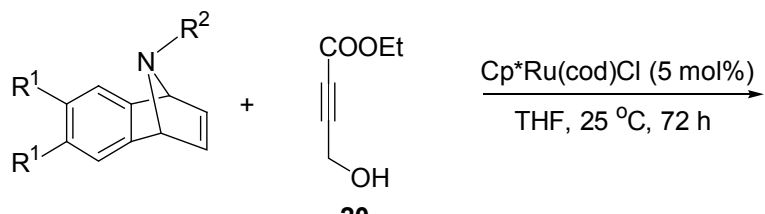<smiles>[R]COC(=O)C1=C(CO)C2C3C(CO)=C(CO)C(c4cc([R])c([R])cc43)N1[R]N2[R2]</smiles>

表 $4 \mathrm{Ru}$ 催化氮杂二环烯烃与炔烃 $\mathbf{2 0}$ 的开环反应

Table 4 Ru-Catalyzed ring-opening reactions of azabicyclic alkenes with alkyne $\mathbf{2 0}$

\begin{tabular}{lllcc}
\hline \multirow{2}{*}{ Entry } & \multirow{2}{*}{$\mathrm{R}^{1}$} & \multicolumn{2}{c}{$\mathrm{R}^{2}$} & \multicolumn{2}{c}{ Yield/\% } \\
\cline { 4 - 5 } & & & $\mathbf{2 1}$ & $\mathbf{2 2}$ \\
\hline 1 & $\mathrm{H}$ & $\mathrm{Boc}$ & 78 & 0 \\
2 & $\mathrm{Br}$ & $\mathrm{Boc}$ & 5 & 0 \\
3 & $\mathrm{H}$ & $\mathrm{CO}_{2} \mathrm{Me}$ & 77 & 0 \\
4 & $\mathrm{H}$ & $\mathrm{CO}_{2} \mathrm{Bn}$ & 80 & 0 \\
5 & $\mathrm{H}$ & $\mathrm{C}(\mathrm{O}) \mathrm{Bu}-t$ & 0 & 82 \\
6 & $\mathrm{H}$ & $\mathrm{Ts}$ & 0 & Trace \\
\hline
\end{tabular}

2008 年, Tenaglia 等 ${ }^{[27]}$ 以富电子结构的 CpRuI$\left(\mathrm{PPh}_{3}\right)_{2}$ 催化氮杂二环烯烃与炔烃的反应(Eq. 12), 反应 得到开环产物 27 和环加成产物 28. 研究表明: 两种产 物的比例受炔烃的结构影响极大，当炔烃为对称炔烃 时，大部分反应得到的是开环产物 27, 产率高达 98\%; 当炔烃为不对称炔烃且 $\mathrm{R}^{2}$ 为极性很大的基团时都能得 


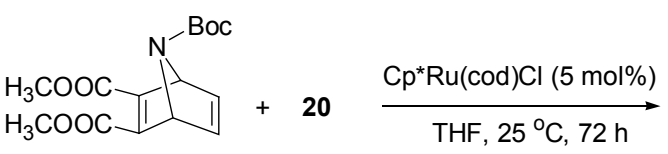

23

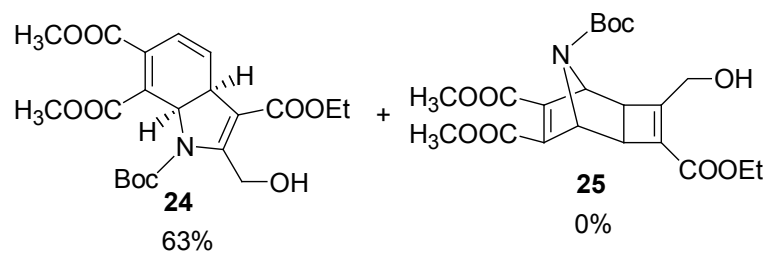

到单一的产物 27, 但是如果取代基含有苯基, 反应只能 得到产物 28(表 5).

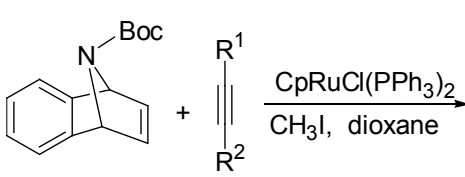

26

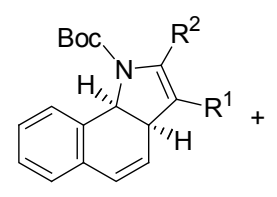

27<smiles>[R]C1=C([R])C2CC1C1c3ccccc3N(C(=O)OC(C)(C)C)C21</smiles>

表 5 炔烃取代基对 $\mathrm{Ru}$ 催化氮杂二环烯烃开环反应的影响

Table 5 Effect of substituents of alkynes on Ru-catalyzed ring-opening reactions

\begin{tabular}{cllll}
\hline \multirow{2}{*}{ Entry } & & \multicolumn{2}{c}{$\mathrm{R}^{1}$} & \multicolumn{2}{c}{$\mathrm{R}^{2}$} & \multicolumn{2}{c}{ Yield $\%$} \\
\cline { 3 - 5 } 1 & $\mathrm{Ph}$ & $\mathrm{Ph}$ & - & $\mathbf{2 8}$ \\
2 & $\mathrm{CH}_{2} \mathrm{OH}$ & $\mathrm{CH}_{2} \mathrm{OH}$ & 88 & - \\
3 & $\mathrm{CH}_{2} \mathrm{OBn}$ & $\mathrm{CH}_{2} \mathrm{OBn}$ & 98 & - \\
4 & $\mathrm{CH}_{2} \mathrm{OMOM}$ & $\mathrm{CH}_{2} \mathrm{OMOM}$ & 82 & - \\
5 & $\mathrm{CH}_{2} \mathrm{OTBDMS}$ & $\mathrm{CH}_{2} \mathrm{OTBDMS}$ & 98 & - \\
6 & $\mathrm{Me}$ & $\mathrm{CH}_{2} \mathrm{OH}$ & 42 & - \\
7 & $\mathrm{Bu}$ & $\mathrm{CH}_{2} \mathrm{OH}$ & 35 & - \\
8 & $\mathrm{Ph}$ & $\mathrm{C} \mathrm{CPh}$ & - & 83 \\
9 & $\mathrm{Ph}$ & $\mathrm{Ac}^{2}$ & - & 98 \\
10 & $\mathrm{Ph}$ & $\mathrm{CO}_{2} \mathrm{Me}$ & - & 98 \\
\hline
\end{tabular}

\section{2 烯烃类化合物作为亲核试剂}

2007 年, Cortez 等 ${ }^{[28]}$ 报道了手性 $\mathrm{Ru}$ 催化剂 30 对氮 杂二环烯烃与芳基烯烃的开环反应(Eq. 13). 底物 29 可 以与带有供电子基团的芳基乙烯反应，也可以与带有拉 电子基团的芳基乙烯反应，反应的产物 $e e$ 值达到 $98 \%$ 以上(表 6).

2007 年, Avenoza 等 ${ }^{[29]}$ 报道了 Hoveyda-Grubbs 第二 代催化剂 33 催化氮杂二环烯烃的研究(Eq. 14). 他们使 用缺电子的一取代烯烃作为亲核试剂, 反应具有很好的 区域选择性，当烯烃取代基为 COOEt 时，反应产率可达 到 $93 \%$.

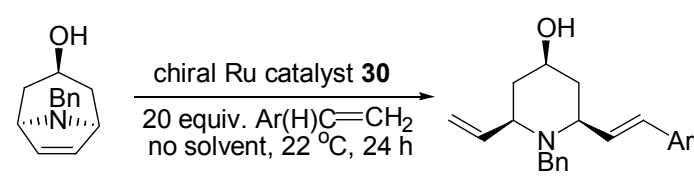

29

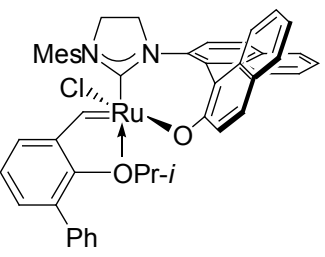

$30 \mathrm{a}$

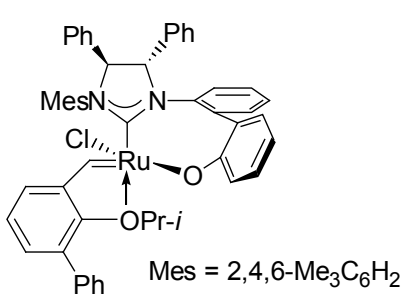

$30 \mathrm{~b}$
表 $6 \mathrm{Ru}$ 催化氮杂二环烯烃 29 与芳香烯烃的开环反应 Table 6 Ru-catalyzed ring-opening reactions of azabicyclic alkenes $\mathbf{2 9}$ with aromatic alkenes

\begin{tabular}{clcccc}
\hline Entry & \multicolumn{1}{c}{$\mathrm{Ar}$} & $\mathrm{Ru}$ catalyst & Conv./\% & Yield/\% & $e e / \%$ \\
\hline 1 & $\mathrm{C}_{6} \mathrm{H}_{5}$ & 30a & $>98$ & 82 & $>98$ \\
2 & $\mathrm{C}_{6} \mathrm{H}_{5}$ & $\mathbf{3 0 b}$ & 80 & 65 & 94 \\
3 & $o-\mathrm{BrC}_{6} \mathrm{H}_{4}$ & $\mathbf{3 0 a}$ & 63 & 55 & 97 \\
4 & $o-\mathrm{BrC}_{6} \mathrm{H}_{4}$ & $\mathbf{3 0 b}$ & 27 & - & - \\
5 & $o-\mathrm{MeC}_{6} \mathrm{H}_{4}$ & $\mathbf{3 0 a}$ & $>98$ & 81 & 98 \\
6 & $o-\mathrm{MeC}_{6} \mathrm{H}_{4}$ & $\mathbf{3 0 b}$ & 95 & 60 & 98 \\
7 & $o-\mathrm{FC}_{6} \mathrm{H}_{4}$ & $\mathbf{3 0 a}$ & 87 & 70 & $>98$ \\
8 & $p-\mathrm{MeOC}_{6} \mathrm{H}_{4}$ & $\mathbf{3 0 a}$ & 87 & 67 & $>98$ \\
9 & $p-\mathrm{MeOC}_{6} \mathrm{H}_{4}$ & $\mathbf{3 0 b}$ & 50 & 38 & 96 \\
10 & $p-\mathrm{CF}_{3} \mathrm{C}_{6} \mathrm{H}_{4}$ & $\mathbf{3 0 a}$ & $>98$ & 78 & 97 \\
\hline
\end{tabular}

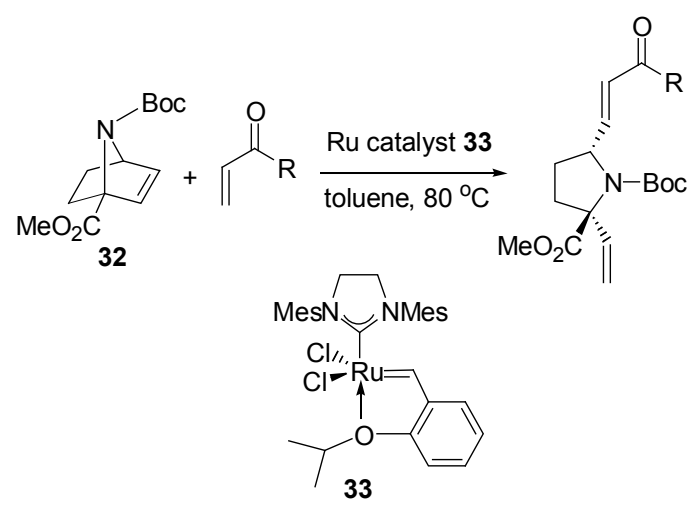

\section{4 铑催化氮杂二环烯烃的开环反应}

\section{1 胺类化合物作为亲核试剂}

2002 年 Lautens 研究小组 ${ }^{[30]}$ 首次报道了铑催化氮杂 二环烯烃与仲胺类亲核试剂的开环反应(Eq. 15). 研究 发现：添加剂和底物 $\mathrm{N}$ 上取代基 $\mathrm{R}$ 对反应的活性和产物 的产率有着重要的影响, 当 $\mathrm{R}$ 和添加剂分别为 Nosyl 和 $\mathrm{NH}_{4} \mathrm{I}$ 时，开环反应活性最好，产率高达 94\%。这类开环 反应的产物再经过氢化后, 就会得到类似镇痛剂药物 35 的 1,2-二氨基四氢化荟的骨架结构, 因此这是有效合 成此类镇痛剂药物的一种方法. 

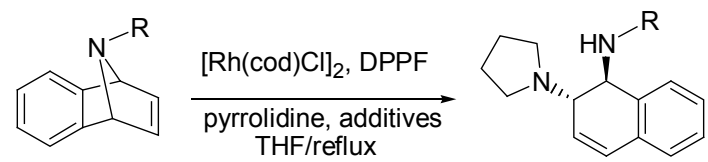<smiles>CN(C(=O)Cc1ccc(Cl)c(Cl)c1)[C@H]1c2ccccc2CC[C@H]1N1CCCC1</smiles>

35

2006 年, Lautens 等 ${ }^{[31]}$ 还对芳香伯胺亲核试剂和底 物芳环上取代基对开环反应的影响进行了研究(Eq. 16), 结果表明: 芳香伯胺作为亲核试剂, 反应效果很好, ee 值高达 97\%; 取代基 $\mathrm{R}^{1}$ 不论是推电子基还是拉电子基, 开环反应活性都很高，产物是 anti 构型，ee 值都在 $94 \%$ 以上，产率可高达 $98 \%$.

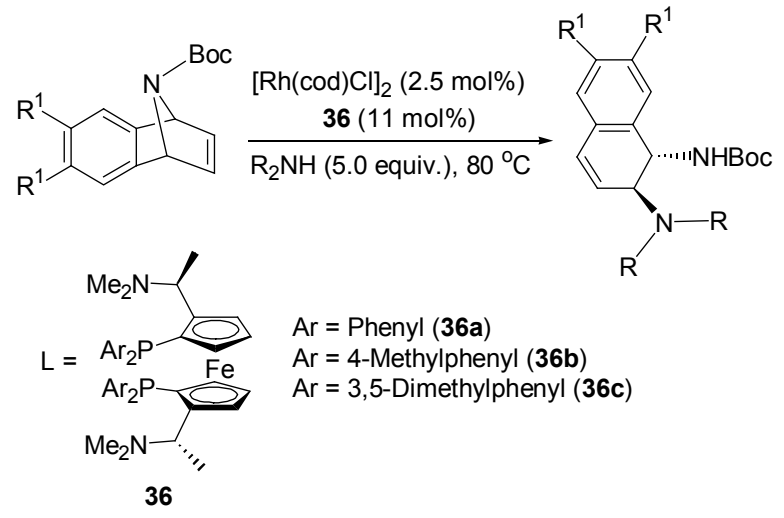

2006 年, Lautens 等 ${ }^{[32]}$ 对铑催化剂的配体和亲核试 剂的用量进行了研究. 研究发现: 反应使用二苠基胺作 为仲胺亲核试剂时, 选择配体 $\mathbf{3 6 b}$, 得到的催化效果最 好; 亲核试剂的用量逐渐从 1.2 equiv. 增至 5 equiv. 时, 反应时间大大缩短, 反应的对映选择性也有所提高, 而 当用量增至 10 equiv., 反应的对映选择性却略有下降.

Lautens 等研究小组还提出了 $\mathrm{Rh}$ 催化氮杂二环烯烃 与胺类亲核试剂的开环反应可能的反应机理 (Scheme 5).

2007 年 ${ }^{[33}$ 和 2010 年 ${ }^{[34]}$ 我们研究小组分别报道了以 哌嗪类化合物为亲核试剂, $[\mathrm{Rh}(\operatorname{cod}) \mathrm{Cl}]_{2}$ 与 $(R, S)-\mathrm{PPF}-\mathrm{P}-$ $(t-\mathrm{Bu})_{2}$ 催化氮杂二环烯烃开环反应, 但是反应的产率和 对映选择性却不如 Lautens 等 ${ }^{[31]}$ 的.

\section{2 有机硼酸类化合物作为亲核试剂}

2007 年, Lautens 等 ${ }^{[35]}$ 研究了 $\mathrm{Rh}$ 催化二氮杂二环烯 烃的开环反应(Eq. 17). 他们尝试使用 $\left[\mathrm{Rh}(\mathrm{CO})_{2} \mathrm{acac}\right]$, $\left[\mathrm{Rh}(\mathrm{CO})_{2} \mathrm{Cl}\right]_{2},\left[\mathrm{Rh}(\mathrm{cod})_{2} \mathrm{Cl}\right]_{2},\left[\mathrm{Rh}(\operatorname{cod})_{2} \mathrm{OH}\right]_{2}$ 等催化剂,



(Selective $\mathrm{C}-\mathrm{N}$ bond cleavage)

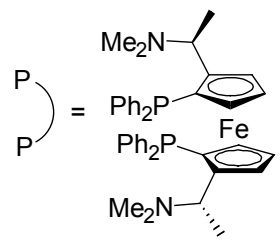

$\left(S, S^{\prime}\right)-\left(R, R^{\prime}\right)-\mathrm{C}_{2}$-Ferriphos

Scheme 5

苯基嗍酸作为亲核试剂在不同的溶剂下对二氮杂二环 化合物进行开环反应的优化，然后把优化条件应用于这 类的开环反应 $(\mathrm{Eq} .17)$. 他们尝试了 $\mathrm{R}$ 基为 $\mathrm{CO}_{2} \mathrm{Et}, \mathrm{Boc}$, $\mathrm{Cbz}$ 和 Troc 的反应，结果反应产率都达到 $91 \%$ 以上.

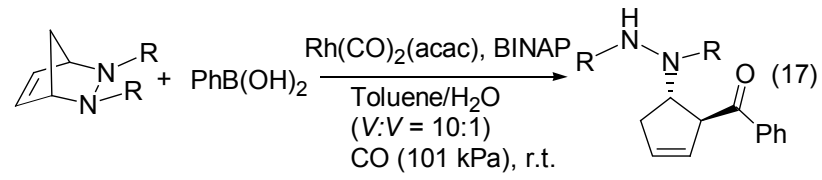

2008 年, Lautens 等 ${ }^{[36]}$ 再次报道了 $\mathrm{Rh}$ 催化二氮杂二 环烯烃的开环反应(Eq. 18). 他们使用 $\left[\mathrm{Rh}(\mathrm{cod})_{2} \mathrm{OH}\right]_{2}$ 和 $t$-Bu-josiphos 作为反应的催化体系, 因为 $t$-Bu-josiphos 配体比 BINAP 配体在 Rh 催化二环体系中具有更好的反 应活性和对映立体选择性. 当亲核试剂为富电子的芳基 硣酸时, ee 值都大于 $96 \%$ (表 7).

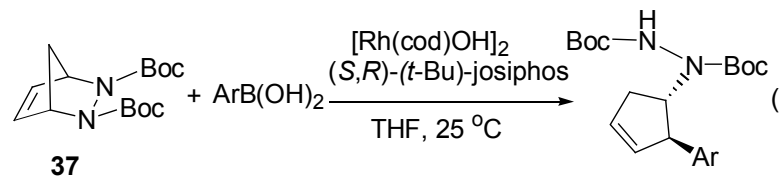

\section{3 末端炔烃类化合物作为亲核试剂}

2008 年, Nishimura 等 ${ }^{[37]}$ 首次报道了 $\mathrm{Rh}$ 催化氮杂二 环烯烃与末端炔烃类化合物的开环反应研究(Eq. 19). 
表 $7 \mathrm{Rh}$ 催化氮杂二环烯烃与芳基苯嗍酸的开环反应

Table 7 Rh-catalyzed ring-opening reactions of diazabicyclic alkenes with aryl boronic acids

Entry

研究发现，末端炔烃取代基 $\mathrm{R}$ 对产率和对映选择性影响 都很大, 当 $\mathrm{R}$ 为空间位阻较大的基团 $\mathrm{Si}(i-\mathrm{Pr})_{3}$ 时, 反应 得到较好的结果(表 8).

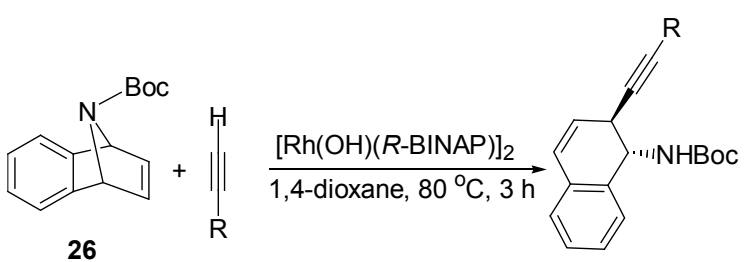

表 8 取代基 $\mathrm{R}$ 对 $\mathrm{Rh}$ 催化 $N$-Boc-氮杂二环烯烃开环反应的影响 Table 8 Effect of $\mathrm{R}$ group on the rhodium-catalyzed ringopening reactions of $N$-Boc-azabicyclic alkenes

\begin{tabular}{|c|c|c|c|}
\hline Entry & $\mathrm{R}$ & Yield $/ \%$ & $e e / \%$ \\
\hline 1 & $\mathrm{C}_{6} \mathrm{H}_{13}$ & 15 & 3 \\
\hline 2 & $\mathrm{SiEt}_{3}$ & 58 & 36 \\
\hline 3 & $\mathrm{Si}(i-\mathrm{Pr})_{3}$ & 67 & 82 \\
\hline 4 & $t-\mathrm{BuOSiMe}_{2}(t-\mathrm{Bu})$ & 81 & 86 \\
\hline
\end{tabular}

他们还探讨了不同配体对开环反应的影响，使用配 体 39a 39e 反应产率可达 49\% 94\%, ee 值为 $77 \%$ $99 \%$. 值得注意是, $\left[\mathrm{Rh}(\mathrm{OAc})\left(\mathrm{C}_{2} \mathrm{H}_{4}\right)\right]_{2}$ 与配体 $(R)-\mathrm{DTBM}-$ segphos 配位形成的催化剂催化效果很好, 反应的产率 高达 93\%, ee 值也高达 99\% (Eq. 20).
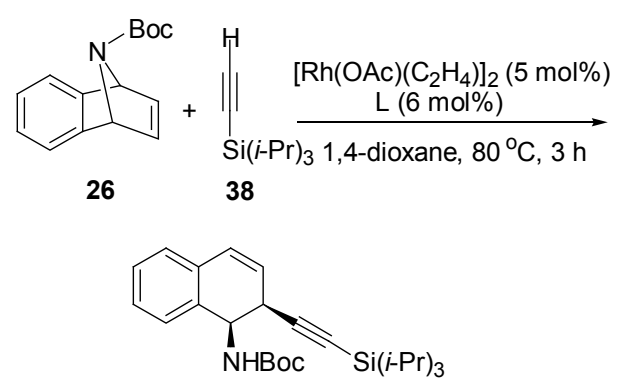

40

$93 \%$ yield, $99 \%$ ee



(R)-binap $39 a$



(R)-MeO-biphep

$39 d$



(R)- $\mathrm{H}_{8}$-binap $39 \mathrm{~b}$<smiles>Pc1ccccc1-c1c(-c2ccccc2)ccc2c1OCO2</smiles>

$(R)$-segphos $39 \mathrm{c}$
他们的研究还表明: 底物芳环上取代基对开环反应 的影响不大. 不管芳环上取代基是拉电子基团还是推电 子基团，反应均能得到相应的开环产物，并且产率高达 $83 \% \sim 94 \%$, 对映选择性也很好, ee 值高达 $98 \%$ 以上.

\section{5 铱催化氮杂二环烯烃的开环反应}

2008 年, 我们研究小组 ${ }^{[38]}$ 首次以 $[\operatorname{Ir}(\operatorname{cod}) \mathrm{Cl}]_{2}$ 与 (S)-BINAP 双膦配体形成的配合物作催化剂, 以仲胺作 亲核试剂, 成功实现了 $N$-Boc-氮杂二环烯烃的开环反 应(Eq. 21), 开环反应的产物的产率可达 $86 \%$, ee 值为 $87 \%$. 我们研究发现 $N$-取代哌嗪仲胺类亲核试剂能得到 令人满意的结果, 并且苯基上连有给电子基比连有拉电 子基反应活性高，而 $N$-甲基苯胺亲核试剂效果不是很 好, 这是因为 $\mathrm{N}$ 上的孤对电子与苯环大 $\pi$ 键电子发生 $\mathrm{p}-\pi$ 共轭效应，降低了亲核试剂的亲核活性. 有趣的是, 在反应体系中加入卤化铵类添加剂却不利于开环反应, 这与我们先前报道 ${ }^{[33]}$ 的卤化铵类添加剂在 $\mathrm{Rh}$ 催化氮杂 二环烯烃开环反应中的作用相反. 
<smiles>CC(C)(C)OC(=O)N1c2ccccc2C2C=CC21</smiles>

26<smiles>[R]c1ccc(N2CCN([C@@H]3C=Cc4ccccc4C3NC(=O)[O-])CC2)cc1</smiles>

2010 年, 我们研究小组 ${ }^{[39]}$ 再次报道了铱催化氮杂 二环烯烃与伯胺的开环反应(Eq. 22). 当伯胺作为亲核 试剂时, 我们发现底物 $\mathrm{N}$ 原子上的取代基对开环反应产 物的对映选择性有着显著的影响, 例如当亲核试剂都是 对溴苯胺时, $\mathrm{R}$ 为 Boc 产物 $e e$ 值高达 $83 \%$, 而 $\mathrm{R}$ 为 $\mathrm{Ts}$ 时, $e e$ 值却只有 $22 \%$; 当亲核试剂苯环上含有富电子基 团时, 反应可得到高产率(96\%)和较好的 $e e$ 值 $(97 \% e e)$; 但苯环上含有拉电子基团时, 反应产率和 $e e$ 值减小(表 9). 我们还进一步提出了开环反应可能的机理(Scheme $6)$.

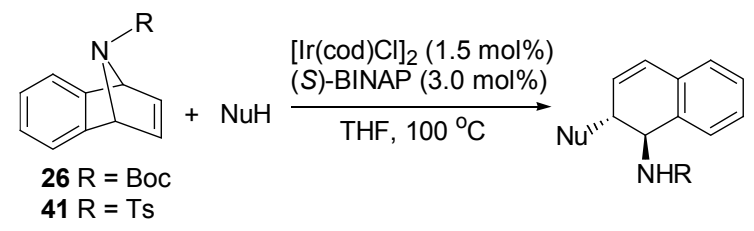

表 9 Ir 催化氮杂二环烯烃与芳香伯胺亲核试剂的开环反应

Table 9 Iridium-catalyzed ring-opening reactions of azabicyclic alkenes with primary aromatic amine Nucleophiles

\begin{tabular}{|c|c|c|c|c|}
\hline Entry & Substrate & $\mathrm{NuH}$ & Yield/\% & $e e / \%$ \\
\hline 1 & 26 & & 79 & 83 \\
\hline 2 & 26 & & 58 & 86 \\
\hline 3 & 41 & & 96 & 94 \\
\hline 4 & 41 & & 58 & 97 \\
\hline 5 & 41 & & 72 & 94 \\
\hline 6 & 41 & & 22 & 22 \\
\hline
\end{tabular}

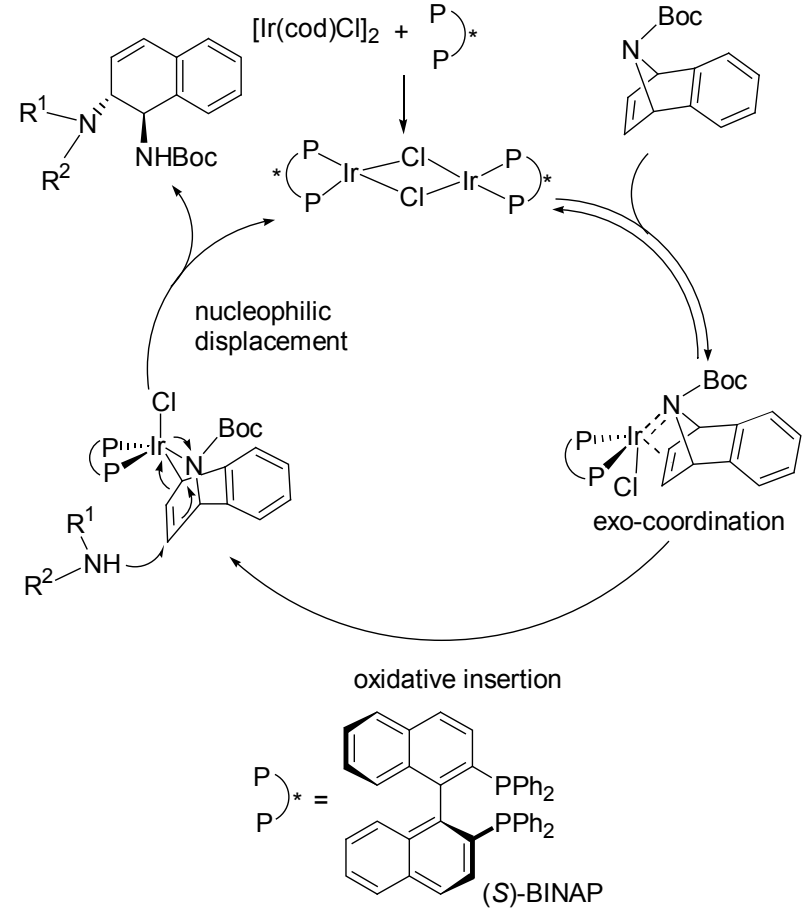

Scheme 6

\section{6 钯催化氮杂二环烯烃的开环反应}

\section{1 有机金属化合物作为亲核试剂}

有机锌试剂作为亲核试剂，易制备，活泼性适中， 价格也不贵. 最常用的是二烷基锌试剂，该类试剂整个 分子反应活性非常低, 但是, 在手性配体中的影响下, $\mathrm{R}_{2} \mathrm{Zn}$ 的分子发生极化，大大提高了其亲核活性. 适中的 活泼性使其在不对称催化反应中具有较弱的背景效应, 因而该类试剂是在不对称催化加成反应领域的常用亲 核试剂.

2000 年, Lautens 等 ${ }^{[40]}$ 首次将有机锌试剂用于 Pd 催 化的氮杂二环烯烃的开环反应(Eq. 23). 研究表明: 他们 将优化后的最佳实验条件用于氮杂二环烯烃的开环反

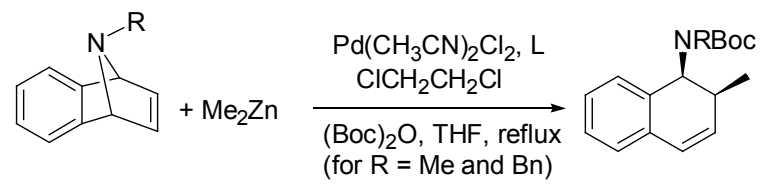

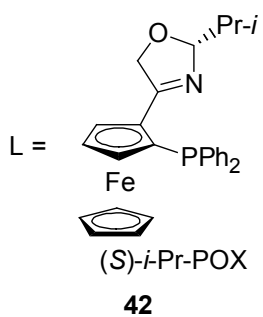


应，发现反应的产率及 $e e$ 值都有很大程度下降，如果将 先前的常温反应改为在加热回流条件下进行, 就能得到 令人满意的结果; 底物 $\mathrm{N}$ 上取代基 $\mathrm{R}$ 对产率影响较大, 而对对映选择性影响较小, 当 $\mathrm{R}$ 为具有拉电子效应的苯 基时, 更有利于开环反应. 产率高达 99\%, ee 值高达 $99 \%$ 以上.

2005 年, Cabrera 等 ${ }^{[41]}$ 报道了一种 $\mathrm{P} / \mathrm{S}$ 配体与 $\mathrm{Pd}$ 配 合的新型高效催化剂, 该催化剂在空气中很稳定. 他们 将它应用于有机锌与氮杂二环烯烃的开环反应，产率可 达 $73 \%$, ee 值在 $99 \%$ 以上(Eq. 24).<smiles>C=CC1c2ccccc2N1[As]</smiles>

41
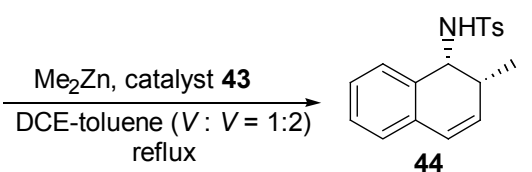

$73 \%$ yield, > $99 \%$ ee

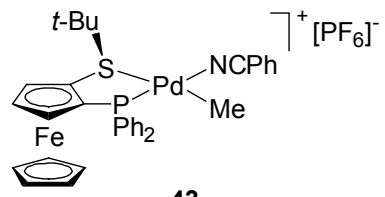

43

2009 年, Ogura 等 ${ }^{[42]}$ 尝试以双钯配合物作催化剂, 催化有机锌亲核试剂与氧杂二环烯烃的开环反应, 并将 最佳反应条件应用于氮杂二环烯烃的开环反应(Eq. 25), 发现 AgOTf 以 $6.6 \mathrm{~mol} \%$ 的量作为添加剂加入最有利于 提高产物的 $e e$ 值, 他们还研究了底物芳环上取代基 $\mathrm{R}^{1}$ 和 $\mathrm{N}$ 上取代基 $\mathrm{R}^{2}$ 对反应的影响, 结果表明, $e e$ 值在很大 程度上取决于 $\mathrm{R}^{1}$, 而受 $\mathrm{R}^{2}$ 影响较小 (表 10 ), 这与 Lautens 等 ${ }^{[30]}$ 报道的胺类亲核试剂与氮杂二环烯烃的开 环反应所得结果一致. 所得的开环反应产物均为 $s y n$ 构 型, $e e$ 值为 $72 \% \sim 99 \%$.

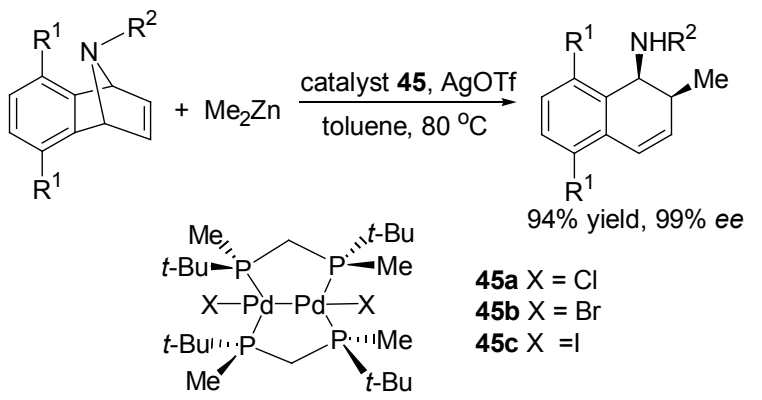

2007 年, Radhakrishnan 等 ${ }^{[43]}$ 把有机铟试剂应用于 钯催化氮杂二环烯烃的开环反应(Eq. 26). 有机铟试剂 与其他的有机金属试剂相比, 有以下优点: 对杂原子亲 核性低; 由于不受空气中氧气的影响，反应易于操作； 没有毒副作用. 他们研究出最佳反应条件后, 进行了一 系列钯催化氮杂二环烯烃开环反应(表 11).
表 $10 R^{1}$ 和 $R^{2}$ 对双 $P d$ 催化氮杂二环烯烃的开环反应的影响 Table 10 Effect of $R^{1}$ and $R^{2}$ groups on the double palladium-catalyzed ring-opening reactions of azabicyclic alkenes

\begin{tabular}{cllcc}
\hline Entry & \multicolumn{1}{c}{$\mathrm{R}^{1}$} & \multicolumn{1}{c}{$\mathrm{R}^{2}$} & Yield $/ \%$ & $e e / \%$ \\
\hline 1 & $\mathrm{H}$ & $\mathrm{Ts}$ & 88 & 72 \\
2 & $\mathrm{Me}$ & $\mathrm{Ts}$ & 79 & 88 \\
3 & $\mathrm{MeO}$ & $\mathrm{Ts}$ & 94 & 99 \\
4 & $\mathrm{BnO}$ & $\mathrm{Ts}$ & 91 & 95 \\
5 & $\mathrm{MOMO}$ & $\mathrm{Ts}$ & 88 & 74 \\
6 & $\mathrm{MeO}$ & $\mathrm{PhSO}_{2}$ & 83 & 99 \\
7 & $\mathrm{MeO}$ & $4-\mathrm{O}_{2} \mathrm{NC}_{6} \mathrm{H}_{4} \mathrm{SO}_{2}$ & 74 & 99 \\
8 & $\mathrm{MeO}$ & $\mathrm{Ms}$ & 75 & 98 \\
9 & $\mathrm{MeO}$ & $\mathrm{Ts}$ & 72 & 80 \\
10 & $\mathrm{BnO}$ & $\mathrm{Ts}$ & 70 & 91 \\
\hline
\end{tabular}

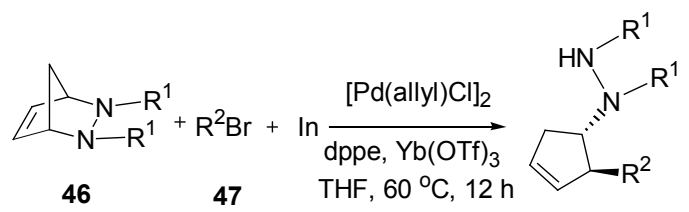

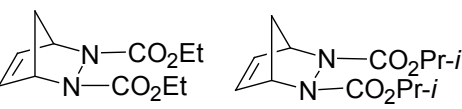

$46 a$<smiles>O=C(O)[R8](Br)(Br)/C=C/CBr</smiles>

46d
$47 a$

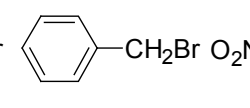



$46 c$
表 11 钯催化氮杂二环烯烃与有机铟的开环反应

Table 11 Palladium-catalyzed ring-opening reactions of azabicyclic alkenes with organoindium reagents

\begin{tabular}{cccc}
\hline Entry & Substrate & Halide & Yield/\% \\
\hline 1 & $\mathbf{4 6 a}$ & $\mathbf{4 7 a}$ & 95 \\
2 & $\mathbf{4 6 b}$ & $\mathbf{4 7 a}$ & 86 \\
3 & $\mathbf{4 6 c}$ & $\mathbf{4 7 a}$ & 88 \\
4 & $\mathbf{4 6 d}$ & $\mathbf{4 7 a}$ & 80 \\
5 & $\mathbf{4 6 a}$ & $\mathbf{4 7} \mathbf{b}$ & 89 \\
6 & $\mathbf{4 6 b}$ & $\mathbf{4 7} \mathbf{b}$ & 80 \\
7 & $\mathbf{4 6 c}$ & $\mathbf{4 7} \mathbf{b}$ & 75 \\
8 & $\mathbf{4 6 d}$ & $\mathbf{4 7} \mathbf{b}$ & 72 \\
9 & $\mathbf{4 6 a}$ & $\mathbf{4 7 c}$ & 43 \\
10 & $\mathbf{4 6 a}$ & $\mathbf{4 7 c}$ & 35 \\
\hline
\end{tabular}

2010 年, Radhakrishnan 等 ${ }^{[44]}$ 成功地把有机镓试剂 应用于钯催化氮杂二环烯烃的开环反应中(Eq. 27), 由 于有机镓试剂反应活性没有有机铟试剂反应活性高，所 以该反应时间更长，而且产率也有所减少. 他们还提出 了该类型反应的反应机理(Scheme 7).

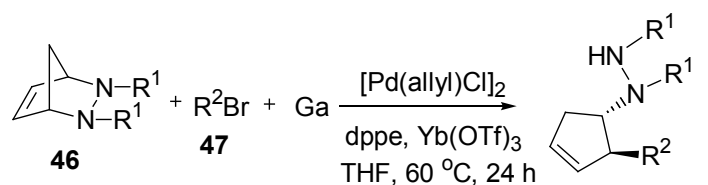




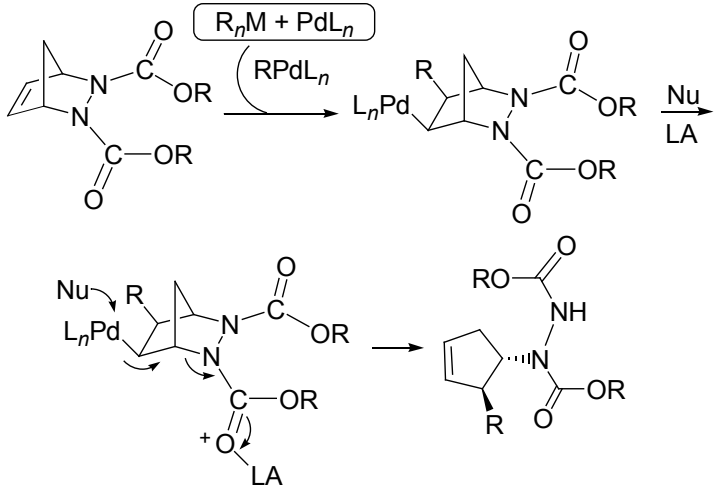

Scheme 7

\section{2 芳香嗍酸类化合物作为亲核试剂}

2003 年, Lautens 等 ${ }^{[45]}$ 用芳香硼酸类化合物作为亲 核试剂, 成功地实现了在氮杂二环烯烃上引进芳基(Eq. 28), 其中以苯基硼酸作为亲核试剂所得产率最高. 研 究发现, 配体为 1,3-二(二苯基膦)丙烷(dppp), 溶剂为 $\mathrm{MeOH}$, 可提高反应速率. 另外 $\mathrm{Cs}_{2} \mathrm{CO}_{3}$ 作为添加剂能进 一步提高反应速率，开环反应转化率为 $99 \%$.

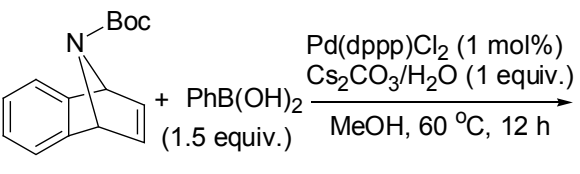

26

\section{3 磑代芳烃化合物作为亲核试剂}

2006 年, Chen 等 ${ }^{[46]}$ 报道了 Pd 催化氧杂二环烯烃的 开环反应. 他们研究发现以 2.2 倍催化剂量的三苯基膦 $\left(\mathrm{PPh}_{3}\right)$ 作配体，二甲基甲酰胺(DMF)为溶剂，室温条件 下, 开环反应效果最好. 在此反应中，1,2,2,6,6-五甲基 哌啶(PMP) 和 $\mathrm{Zn}$ 作添加剂是必要的. 他们将所得最佳反 应条件应用于氮杂二环烯烃的开环反应(Eq. 29), 也取 得了很好的效果. 得到的产物具有很好的立体选择性, 以 anti 构型为主，产率高达 $96 \%$.



1

49<smiles>CCOC(=O)c1ccc([C@H]2C=Cc3ccccc3[C@H]2NC(C)=O)cc1</smiles>

50

2007 年, Cheng 等 ${ }^{[47]}$ 在 $\mathrm{Zn}$ 粉存在下, 也用碘代苯作 为亲核试剂, 在 $\mathrm{Pd}\left(\mathrm{PPh}_{3}\right)_{2} \mathrm{Cl}_{2}$ 催化下, 也实现了氮杂二
环烯烃的开环反应(Eq. 30). 与 Chen 等 ${ }^{[46]}$ 的报道不同的 是，他们得到的产物以 $s y n$ 构型为主，产率也可达 66\%.

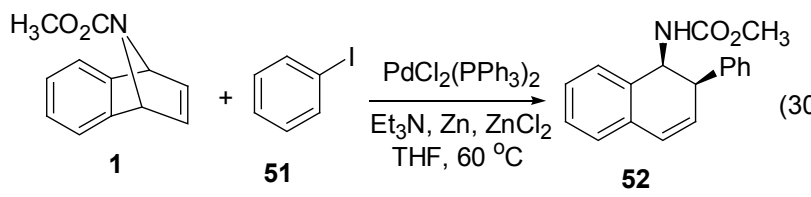

\section{7 结论与展望}

过渡金属催化氮杂二环烯烃的开环反应，是一步合 成具有两个手性中心的化合物的有效途径，同时，也是 合成具有生物活性药物的一种有效方法，因此，该类反 应具有广阔的发展前景和科学意义，过渡金属 $\mathrm{Ni}, \mathrm{Cu}$, $\mathrm{Ru}, \mathrm{Rh}, \mathrm{Ir}$ 和 $\mathrm{Pd}$ 催化氮杂二环烯烃开环反应的立体选择 性、对映选择性均取得了很大进展，尽管已经合成出来 了多种有效的过渡金属配合物催化剂应用于开环反应， 但没有任何一种是适用于所有底物的，因此，发展高立 体选择性、高对映选择性、高催化活性的过渡金属配合 物催化剂，实现高效选择性的开环反应，探索更适合的 反应条件及研究反应机理仍然是将来需要研究的重点 课题之一.

\section{References}

[1] Scott, E. S.; Felix, A. A.; Ratna, C.; David, E. N.; Val, J. W.; Richard, B. M. J. Med. Chem. 1995, 38, 2395.

[2] Roberto, P.; Francesco, B.; Nicola, C. A.; Marcello, L.; Vincenzo, T.; Francesco, F.; Vincenzo, O.; Alberto, G.; Stefano, G. J. Med. Chem. 1995, 38, 942.

[3] Hallinan, K. O.; Honda, T. Tetrahedron 1995, 51, 12211.

[4] Kamal, A.; Gayatri, N. L. Tetrahedron Lett. 1996, 37, 3359.

[5] Sobti, A.; Kim, K.; Sulikowski, G. A. J. Org. Chem. 1996, 61, 6.

[6] Johnson, B. M.; Chang, P. T. L. Anal. Profiles Drug Subst. Excipients 1996, 24, 443.

[7] Murakami, M.; Lgawa, H. Chem. Commun. 2002, 4, 390.

[8] Degnan, A. P.; Meyers, A. I. J. Org. Chem. 2000, 65, 3503.

[9] Wu, M.-S.; Jeganmohan, M.; Cheng, C.-H. J. Org. Chem. 2005, 70, 9545.

[10] Parthasarathy, K.; Jeganmohan, M.; Cheng, C.-H. Org. Lett. 2006, 8,621 .

[11] Alexakis, A.; Hajjaji, S. E.; Polet, D.; Rathgeb, X. Org. Lett. 2007, 9, 3393.

[12] Lautens, M.; Fagnou, K. Proc. Natl. Acad. Sci. U. S. A. 2004, 101, 5455.

[13] Rayabarapu, D. K.; Cheng, C.-H. Acc. Chem. Res. 2003, 36, 48.

[14] Lautens, M.; Fagnou, K.; Hiebert, S. Acc. Chem. Res. 2003, 36, 48.

[15] Feng, C.-C.; Nandi, M.; Sambaiah, T.; Cheng, C.-H. J. Org. Chem. 1999, 64, 3538.

[16] Rayabarapu, D. K.; Shukla, P.; Cheng, C.-H. Org. Lett. 2003, 5, 4903.

[17] Rayabarapu, D. K.; Chiu, C.-F.; Cheng, C.-H. Org. Lett. 2002, 4, 1679.

[18] Rayabarapu, D. K.; Cheng, C.-H. Chem. Eur. J. 2003, 9, 3164.

[19] Wu, M.-S.; Rayabarapu, D. K.; Cheng, C.-H. J. Org. Chem. 2004, $69,8407$. 
[20] Li, L.-P.; Rayabarapu, D. K.; Nandi, M.; Cheng, C.-H. Org. Lett. 2003, 5, 1621 .

[21] Lautens, M.; Fagnou, K. Tetrahedron 2001, 57, 5067.

[22] Arrayás, R. G.; Cabrera, S.; Carreterro, J. C. Org. Lett. 2005, 7, 219.

[23] Arrayás, R. G.; Cabrera, S.; Carreterro, J. C. Synthesis 2006, 1205.

[24] Pineschi, M.; Moro, F. D.; Crotti, P.; Macchia, F. Org. Lett. 2005, 7, 3605 .

[25] Villeneuve, K.; Tam, W. J. Am. Chem. Soc. 2006, $128,3514$.

[26] Burton, R. R.; Tam, W. Org. Lett. 2007, 9, 3287.

[27] Tenaglia, A.; Marc, S. J. Org. Chem. 2008, 73, 1397.

[28] Cortez, G. A.; Baxter, C. A.; Schrock, R. R.; Hoveyda, A. H. Org. Lett. 2007, 9, 2871.

[29] Carreras, J.; Avenoza, A.; Busto, J. H.; Peregrina, J. M. Org. Lett. 2007, 9, 1235.

[30] Lautens, M.; Fagnou, K.; Zunic, V. Org. Lett. 2002, 4, 3465.

[31] Cho, Y.-H.; Zunic, V.; Senboku, H.; Olsen, M.; Lautens, M. J. Am. Chem. Soc. 2006, 128, 6837.

[32] Cho, Y.-H.; Fayol, A.; Lautens, M. Tetrahedron: Asymmetry 2006, $17,416$.

[33] Xie, L.; Yang, D.-Q.; Zhao, S.-Q.; Wang, H.; Liang, L.-H.; Luo, R.-S. Chem. Lett. 2007, 18, 127.

[34] Long, Y.-H.; Yang, D.-Q.; Zeng, H.-P.; Xie, L.; Wu, L.-H.; Mo, H.-H.; Zuo, X.-J. Chin. J. Chem. 2010, 28, 235.
[35] Menard, F.; Weise, C. F.; Lautens, M. Org. Lett. 2007, 9, 5365.

[36] Menard, F.; Lautens, M. Angew. Chem., Int. Ed. 2008, 47, 2085.

[37] Nishimura, T.; Tsurumaki, E.; Kawamoto, T.; Guo, X.-X.; Hayashi, T. Org. Lett. 2008, 10, 4057.

[38] Yang, D.-Q.; Long, Y.-H.; Wang, H.; Zhang, Z.-M. Org. Lett. 2008, 10, 4723.

[39] (a) Long, Y.-H.; Yang, D.-Q.; Zhang, Z.-M.; Wu, Y.-J.; Zeng, H.-P.; Chen, Y. J. Org. Chem. 2010, 75, 7291.

(b) Yang, D.-Q.; Long, Y.-H.; Wu, Y.-J.; Zuo, X.-J.; Tu, Q.-Q.; Fang, S.; Jiang, L.-S.; Wang, S.-Y.; Li. C.-R. Organometallics 2010, 29, 5936.

[40] Lautens, M.; Hiebert, S.; Renaud, J. Org. Lett. 2000, 2, 1971.

[41] Cabrera, S.; Arrayás, R. G.; Alonso, I.; Carretero, J. C. J. Am. Chem. Soc. 2005, 127, 17938.

[42] Ogura, T.; Yoshida, K.; Yanagisawa, A.; Imamoto, T. Org. Lett. 2009, 11, 2245.

[43] John, J.; Anas, S.; Sajisha, V. S.; Viji, S.; Radhakrishnan, K. V. Tetrahedron Lett. 2007, 48, 7225.

[44] John, J.; Adarsh, B.; Radhakrishnan, K. V. Tetrahedron 2010, 66, 1383.

[45] Lautens, M.; Dockendorff, C. Org. Lett. 2003, 5, 3695.

[46] Chen, C. L.; Martin, S. F. J. Org. Chem. 2006, 71, 4810.

[47] Rayabarapu, D. K.; Cheng, C.-H. Acc. Chem. Res. 2007, 40, 971.

(Zhao, C.) 